\title{
Influence de l'écrouissage sur les propriétés magnétiques d'alliages Fe-3 \%Si non orientés
}

\author{
E. Hug $\left({ }^{1}\right)$, M. Kant $\left({ }^{1}\right)$ et M. Clavel $\left({ }^{2}\right)$
}

(1) Division Electromécanique, Département Génie Mécanique, Centre de Recherches de Royallieu, Université de Technologie de Compiègne, BP 649, 60206 Compiègne Cedex, France (2) Division Mécanique LG2MS, Département Génie Mécanique, Centre de Recherches de Royallieu, Université de Technologie de Compiègne, BP 649, 60206 Compiègne Cedex. France

(Reçu le 14 janvier 1994, accepté le 14 avril 1994)

\begin{abstract}
Résumé. - L'évolution des propriétés magnétiques d'alliages $\mathrm{Fe}-3 \% \mathrm{Si}$ à grains non orientés avec les déformations plastiques a été évaluée à l'aide d'une technique expérimentale permettant des mesures directes sur éprouvettes de traction. Une dégradation importante de ces caractéristiques est observée aux faibles et moyennes amplitudes d'excitation magnétıque. Cet effet s'estompe pour les valeurs les plus élevées du champ magnétique. A mesure qu'augmente la déformation, les dislocations s organisent en cellules quasi hexagonales sur le palier de Lüders, puis, en début d'écrouissage, se regroupent en petits amas faiblement denses séparant des zones de métal où existent des dislocations isolées. Ces deux configurations sont les plus nocives pour les propriétés magnétiques. Aux fortes déformations, des murs de dislocations très denses se forment, mais ne dégradent pas davantage les caractéristiques magnétiques. Nous montrons que le modèle du potentiel conservatif ne s'applique pas à nos résultats expérimentaux tandis que celui de courbure des parois pourrait être mieux adapté. L'évolution du champ coercıtif avec la contrainte plastique pure appliquée au matériau suit une loi en deux stades, contrairement aux trois stades obtenus sur du Fe polycristallin de haute pureté par d'autres auteurs.
\end{abstract}

Abstract. - Magnetic characteristics were measured on stress-strain test pieces of non oriented $3 \% \mathrm{SiFe}$ with a magnetic measurement frame. Plotting various magnetic properties against plastic deformation shows an exponential damage for low and medium values of magnetic field amplitude. This phenomenon is much weaker when high magnetic field amplitudes are reached. Metallurgical state of the material between 0 and $0.5 \%$ is characterized by the Luiders strain state, which involves an important heterogeneous dislocations structure inside the metal die. After this, dislocation structure remains more and less homogeneous from 0 to $10 \%$ strain (small tangles and isolated dislocations). These two dislocations structures are the more harmful for the magnetic properties of the alloys. From $\varepsilon_{\mathrm{p}}=10 \%$ to fracture, dislocations configuration becomes progressively inhomogeneous and walls of high density are created but any supplementary magnetic damage is observed. Potential theories are not able to explain our experimental results but domain wall bowing theories could successfully be applied. Coercive field versus plastic stress displays two distinct linear evolutions against three in the case of polycristalline high-purity iron, as shown by others authors. 


\section{Introduction.}

Les pertes de puissance totales au sein d'un moteur électrique sont souvent très supérieures aux caractéristiques "classiques » du matériau mesurées par un dispositif expérimental normalisé (cadre d'Epstein, SST, . ). Cet écart important pose un problème à la fois aux constructeurs de machines, qui ne disposent pas toujours de données suffisamment fiables sur les matériaux employés dans la conception de leurs produits, et aux laboratoires de recherche qui tentent depuis de nombreuses années de comprendre et de prévoir les pertes de puissance en fonction des différentes sollicitations physiques imposées aux alliages. L'écart entre les mesures normalisées et celles effectuées sur machines électriques peut avoir plusieurs origines comme l'existence de « points chauds » dans les tôles d'une machine en service, modifiant localement leurs caractéristiques magnétiques [1], l'excitation électromagnétique complexe, non sinusoïdale [2], ou encore l'évolution des propriétés magnétiques des matériaux lorsqu'ils sont soumis à des déformations élastiques [3-9], plastiques [10-12] ou lorsqu'ils sont le siège de contraintes internes [13].

L'objectif de ce travail est l'étude des modifications des caractéristiques magnétiques d'alliages ferromagnétiques doux $\mathrm{Fe}-3 \% \mathrm{Si}$ non orientés avec l'écrouissage. Le premier paragraphe est consacré à une présentation du matériau et des moyens expérimentaux mis en œuvre pour cette étude. Nous donnons ensuite des résultats concernant d'une part le comportement mécanique du matériau, d'autre part l'évolution de ses propriétés magnétiques avec les déformations plastiques imposées. Une analyse microscopique de la structure des dislocations complète ces mesures. Nous concluons par une discussion sur les interactions magnétomécaniques entre dislocations et parois de Bloch, dans le cadre des modèles du potentiel conservatif et de courbures des parois.

\section{Méthodologie.}

1. 1 PRÉSENTATION DES Alliages. - L'alliage étudié est un Fe-3,2 \%Si à grains non orientés, se présentant sous la forme de tôles d'épaisseur $0,35 \mathrm{~mm}$. Les essais mécaniques et les mesures magnétiques sont réalisés le long de la direction de laminage DL.

1.2 ESSAIS MÉCANIQUES. - Des essais de traction monotone sont effectués à température ambiante pour imposer un certain taux de déformation plastique $\varepsilon_{\mathrm{p}}$. La vitesse de traction est fixée à une valeur arbitraire. Les éprouvettes subissent au préalable un recuit de détente, effectué sous vide partiel $\left(P_{\mathrm{O}_{2}}=10^{-3}\right.$ torr $)$, à $750^{\circ} \mathrm{C}$ pendant $10 \mathrm{~h}$, afin d'éliminer les contraintes dues à l'usinage. Les composantes isotrope et cinématique de l'écrouissage sont déterminées à l'aide d'essais cycliques de charge-décharge, avec un écrouissage progressif jusqu'à la rupture.

1.3 Mesures magnéTIQUes. - Les propriétés magnétiques des éprouvettes en fonction de la déformation plastique imposée s'obtiennent par des mesures directes sur éprouvettes de traction (Fig. 1) [14]. Le principe consiste à créer un champ magnétique dans une culasse ferromagnétique à l'aide d'un dispositif de bobinages (bobines primaires d'excitation), enroulé autour du corps, et excité par un courant électrique de forme définie. Cette culasse est constituée d'un très grand nombre de tôles d'alliage magnétique doux, découpées en forme de « $U$ » rectangulaire, recuites et collées les unes aux autres. Nous disposons aux extrémités l'éprouvette de traction préalablement déformée. Un système de serrage permet sa fixation et maintient un entrefer faible et constant entre l'échantillon et la culasse. Le système de mesures est conçu de telle sorte que la reluctance du «U» soit toujours négligeable devant celle de l'éprouvette, quelles que soient l'intensité du champ magnétique d'excitation et de la déformation plastique imposée. 


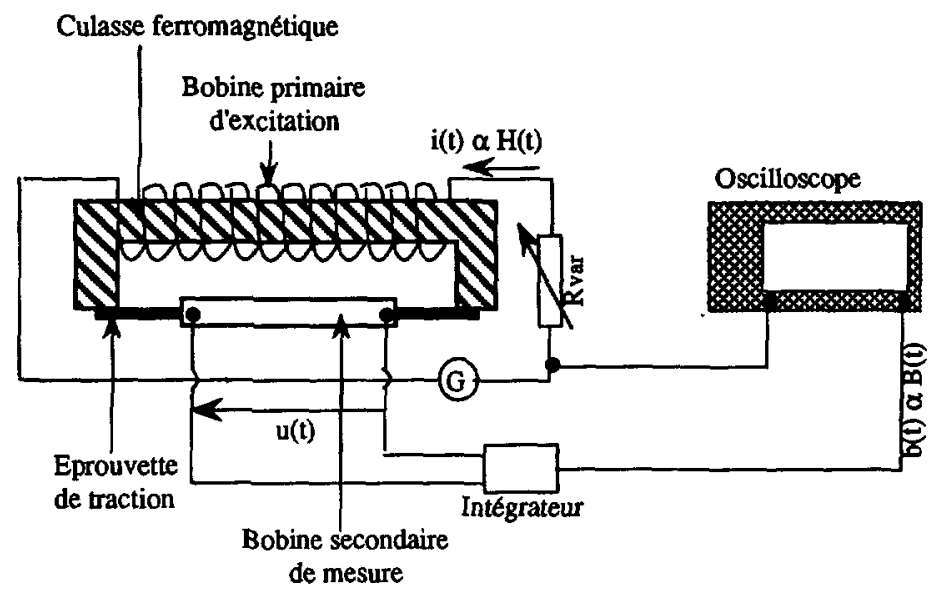

Fig. 1. - Dispositif expérimental de mesure des propriétés magnétiques sur éprouvettes de traction.

[Experimental system for magnetic measurements on stress-strain test pieces.]

Une bobine rectangulaire plate est glissée autour de l'éprouvette pour mesurer la tension induite $u(t)$ due aux variations temporelles de flux magnétique. Cette tension est ensuite traitée par un intégrateur, qui délivre un signal directement proportionnel à l'induction magnétique. La courbe d'aimantation et les pertes massiques de puissance sont mesurées à courant sinusoïdal $i(t)$ imposé, de fréquence $50 \mathrm{~Hz}$. Les signaux $i(t)$ et $b(t)=\int u(t) \mathrm{d} t$ sont recueillis sur un oscilloscope où nous visualisons $b(t)=F(i(t))$, cycle d'hystérésis proportionnel au cycle $B(H)$. Le champ coercitif est obtenu sous des conditions d'excitation quasi statiques.

Les caractéristiques magnétiques initiales du matériau, mesurées sur une éprouvette de traction non déformée, sont comparables aux caractéristiques obtenues par un cadre d'Epstein sollicité par la même excitation magnétique.

1.4 Observations microscopiques. - Parallèlement aux mesures des caractéristiques mécaniques et magnétiques, des lames minces sont réalisées à partir de chaque éprouvette déformée, et examinées par Microscopie Electronique en Transmission (MET). L'évolution de la microstructure avec l'écrouissage est également suivie par microscopie optique.

\section{Résultats expérimentaux.}

2.1 COMPORTEMENT MÉCANiQue DeS alliages Fe-3\%Si.

2.1.1 Ecrouissage monotone. - La courbe de traction conventionnelle $\sigma(\varepsilon)$ est typique d'un acier doux faiblement allié : pic d'élasticité de faible amplitude $\Delta \sigma_{\mathrm{e}}$, existence d'un palier de Lüders de longueur $L_{\mathrm{p}}$ (Fig. 2), puis écrouissage conséquent suivi d'une stagnation à contrainte maximale $\sigma_{\mathrm{m}}$. Le tableau I résume les caractéristiques mécaniques typiques en traction obtenues.

2.1.2 Essais cycliques de charge-décharge. - Après avoir atteint un certain niveau de contrainte, la décharge n'est pas purement élastique, le matériau rentre en compression 


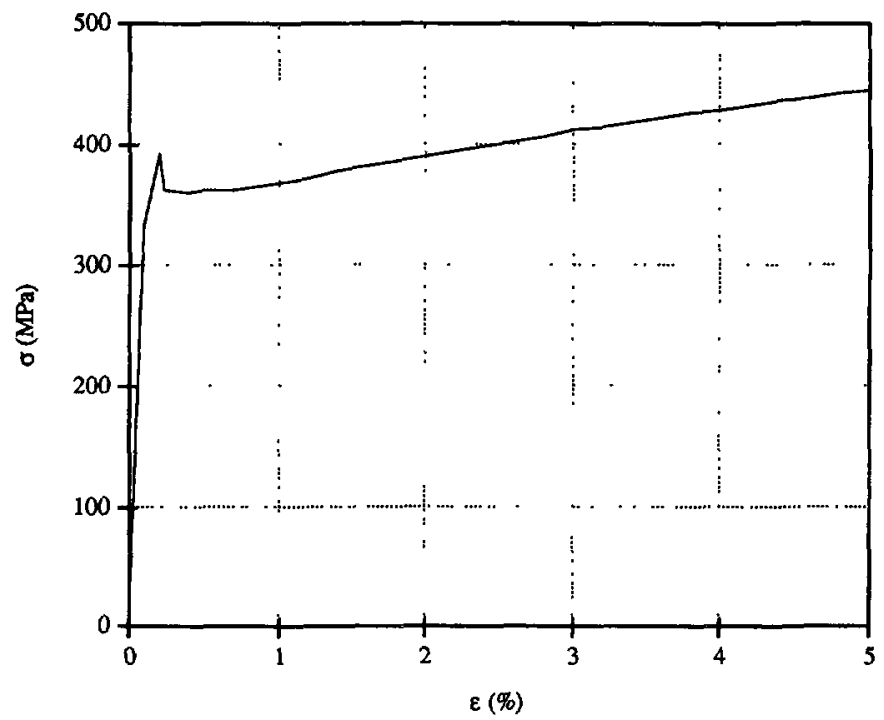

Fig. 2. - Courbe de traction conventionnelle d'un alliage Fe-3\%Si à grains non orienté. Gamme des faibles déformations (Température ambiante, vitesse de déformation: $6,67 \times 10^{-4} \mathrm{~s}^{-1}$ direction de laminage).

[Tensile test characteristic curve of non-oriented $3 \%$ SiFe alloy. Weak deformations (Room-temperature test, strain rate : $6,67 \times 10^{-4} \mathrm{~s}^{-1}$ rolling direction.)]

Tableau I. - Caractéristiques mécaniques moyennes en traction du $\mathrm{Fe}-3 \% \mathrm{Si}$ non orienté dans le sens de laminage, à température ambiante et pour $\mathrm{d} \varepsilon / \mathrm{d} t=6,67 \times 10^{-4} \mathrm{~s}^{-1}$ $\left(\sigma_{\mathrm{e} 0,2}\right.$. limite d'élasticité conventionnelle d̀ $0,2 \%, \Delta \sigma_{\mathrm{e}}=\sigma_{\mathrm{e} \max }-\sigma_{\mathrm{e} \min }, \sigma_{\mathrm{m}}$. contrainte maximale, $A$ : allongement à rupture, $L_{\mathrm{p}}$. longueur $d u$ palier de Lüders).

[Typical mechanical characteristics of tensile testing non-oriented $3 \%$ SiFe. Rolling direction, room-temperature test, strain rate : $6.67 \times 10^{-4} \mathrm{~s}^{-1}\left(\sigma_{\mathrm{e} 02}\right.$. conventional yield stress, $\Delta \sigma_{\mathrm{e}}=\sigma_{\mathrm{e} \max }-\sigma_{\mathrm{e} \text { min }}, \sigma_{\mathrm{m}}$. maximal stress, $A$ : rupture elongation, $L_{\mathrm{p}}$. Lüders state length.]

\begin{tabular}{|c|c|c|c|c|}
\hline$\sigma_{\mathrm{e} 0,2}(\mathrm{MPa})$ & $\Delta \sigma_{\mathrm{e}}(\mathrm{MPa})$ & $\sigma_{\mathrm{m}}(\mathrm{MPa})$ & $A(\%)$ & $L_{\mathrm{p}}(\%)$ \\
\hline 359,4 & 9,4 & 493,8 & 25,6 & 0,18 \\
\hline
\end{tabular}

(Fig. 3a). Ce phénomène permet, en effectuant des essais cycliques de charge-décharge à rupture sur des éprouvettes de traction, d'accéder aux composantes isotrope $R$ et cinématique $X$ de l'écrouissage [14] (Fig. 3b). La figure 4 donne l'évolution de $R$ et $X$ avec $\varepsilon_{\mathrm{p}}$, normalisée par rapport à la contrainte maximale $\sigma_{\mathrm{m}}$. Les décharges sont effectuées tous les pourcents de déformation. L'écrouissage est fortement cinématique : $X$ croît rapidement entre 0 et $10 \%$ de déformation puis se stabilise au-delà ; parallèlement, $R$ chute dès le premier pourcent de déformation puis oscille autour d'une valeur moyenne.

2.1.3 Modèle d'écrouissage. — La vérification du comportement cinématique du matériau est obtenue grâce à une simulation numérique des points expérimentaux par le logiciel SiDoLo [15]. Celui-ci développe un modèle d'écrouissage du type Lemaitre-Chaboche [16], dans 


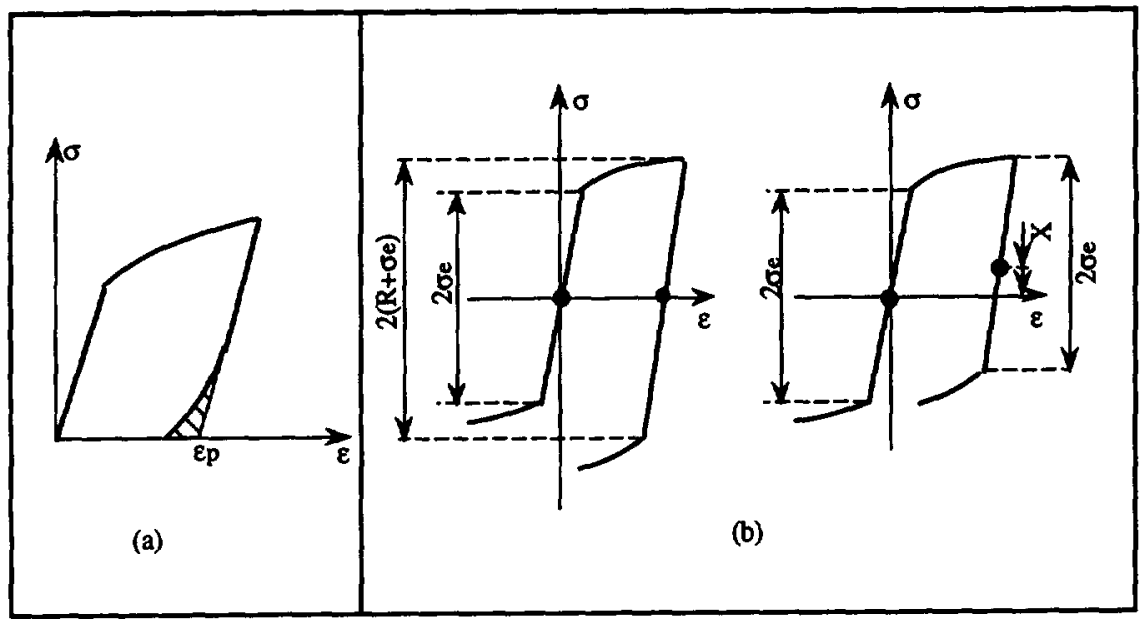

Fig. 3. - (a) Principe d'une microplasticité à décharge ; (b) représentation schématique sur un essai de traction de l'écrouissage isotrope $(R)$ et cinématique $(X)$.

[(a) Microplasticity when unloading test pieces; (b) strain hardening coefficients $R$ and $X$ : schematic representation.]

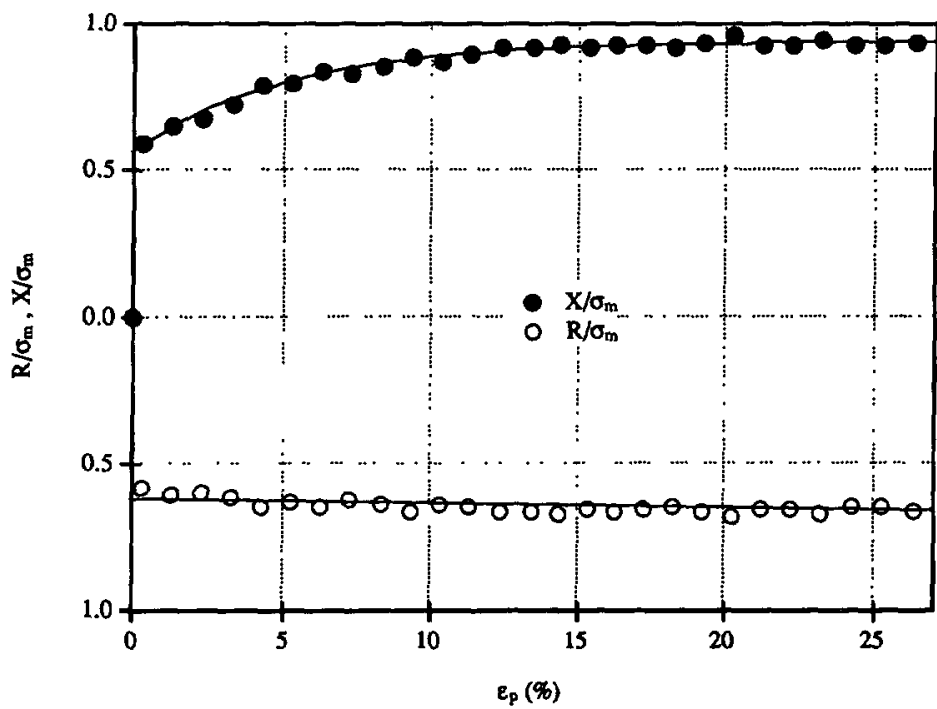

Fig. 4. - Evolution des composantes d'écrouissage isotrope et cinématique normalisées par rapport à la contrainte maximale $\sigma_{\mathrm{m}}$, avec les déformations plastiques.

$\left[R\right.$ and $X$, normalized repect to $\sigma_{\mathrm{m}}$, versus plastic strain.]

lequel l'écrouissage isotrope s'écrit :

$$
R=\frac{q_{\mathrm{r}}}{b_{\mathrm{r}}}\left(1-\mathrm{e}^{-b_{\mathrm{r}} \cdot \varepsilon_{\mathrm{p}}}\right)
$$


$q_{\mathrm{r}}$ et $b_{\mathrm{r}}$ sont des coefficients représentatifs du modèle. L'écrouissage cinématique se met sous la forme :

$$
X=X_{1}+X_{2}
$$

avec

$$
\frac{\mathrm{d} X_{\mathrm{l}}}{\mathrm{d} t}=C_{1} \cdot \frac{\mathrm{d} \varepsilon_{\mathrm{p}}}{\mathrm{d} t}-\gamma \cdot X_{1} \cdot \frac{\mathrm{d} \varepsilon_{\mathrm{p}}}{\mathrm{d} t}
$$

et

$$
X_{2}=C_{2} \cdot \varepsilon_{\mathrm{p}}
$$

$X_{1}$ représente l'écrouissage cinématique non linéaire, $X_{2}$ l'écrouissage cinématique linéaire. $\gamma$ est un terme caractéristique de la non-linéarité, $C_{1}$ et $C_{2}$ des constantes déterminées par la simulation. Le tableau II donne les valeurs des différents coefficients obtenues par simulation des essais de charge-décharge. Celle-ci confirme le caractère fortement cinématique non linéaire de l'écrouissage : $C_{1}$ est en effet très grand devant $C_{2}$.

Tableau II. - Détermination, par simulation numérique d'essais de traction, des coefficients caractéristiques de l'écrouissage du FeSiNO avec E le module d'Young et v le coefficient de Poisson.

[Numerical simulation of loading-unloading tests and determination of strain hardening factors for non-oriented $3 \% \mathrm{SiFe}$ with $E$ Young's modulus and $\nu$ Poisson's coefficient.]

\begin{tabular}{|c|c|c|c|c|c|c|c|}
\hline$E(\mathrm{MPA})$ & $\nu$ & $\sigma_{\mathrm{e}}(\mathrm{MPa})$ & $q_{\mathrm{r}}$ & $b_{\mathrm{r}}$ & $C_{1}$ & $C_{2}$ & $\gamma$ \\
\hline $1,88 \times 10^{5}$ & 0,3 & 374,3 & -6500 & 32 & 11100 & 31 & 36 \\
\hline
\end{tabular}

2.2 EVOLUTION DES PROPRIÉTÉS MAGNÉTIQUES AVEC LA DÉFORMATION PLASTIQUE IMPOSÉE. - Les mesures des propriétés magnétiques en fonction des déformations plastiques imposées conduisent aux résultats expérimentaux suivants.

- Pour une amplitude donnée du champ magnétique d'excitation $H_{\mathrm{m}}$, l'induction maximale $B_{\mathrm{m}}$ atteinte diminue avec $\varepsilon_{\mathrm{p}}$ (Fig. 5a); cette tendance est fortement marquée pour les faibles valeurs de $H_{\mathrm{m}}$ (décroissance exponentielle) puis s'atténue à l'approche de la saturation magnétique (décroissance linéaire).

- La forme du cycle d'hystérésis se modifie sensiblement: celui se «couche » sur l'axe horizontal à mesure qu'augmentent les déformations plastiques.

- L'induction rémanente $B_{\mathrm{r}}$, mesurée après saturation magnétique, est également très sensible à $\varepsilon_{\mathrm{p}}$ (Fig. 5b).

- Les perméabilités relatives évoluent de manière similaire. La variation la plus importante survient autour de la perméabilité relative maximale $\mu_{\mathrm{rm}}$ (diminution de 10800 à 2800 entre 0 et $0,5 \%$ de déformation). La perméabilité relative initiale $\mu_{\mathrm{r} i}$ diminue également sensiblement dès les premiers stades de déformation. Lorsque l'aimantation du matériau tend vers la saturation, $\mu_{\mathrm{r}}$ n'évolue plus que très faiblement.

- La figure 5c montre l'évolution des pertes totales de puissance $P$ en fonction de $\varepsilon_{\mathrm{p}}$, pour différentes valeurs de $B_{\mathrm{m}}$. Nous constatons une très forte augmentation à faible induction (pertes multipliées par 40 environ à $0,5 \mathrm{~T}$ ), puis une atténuation du phénomène quand $B_{\mathrm{m}}$ croît. 
- L'évolution du champ coercitif $H_{c}$ (mesuré après saturation sous une excitation quasi statique) avec $\varepsilon_{\mathrm{p}}$ est représentée figure $5 \mathrm{~d} . H_{\mathrm{c}}$ augmente fortement dès les premiers stades de la déformation. Nous obtenons une loi de proportionnalité du type : $H_{\mathrm{c}} \propto \varepsilon_{\mathrm{p}}^{n}, n$ valant $1 / 4$, aux erreurs expérimentales près. Ce résultat a déjà été obtenu par d'autres auteurs pour un alliage $\mathrm{Fe}-3 \% \mathrm{Si}$ à texture de Goss [17].
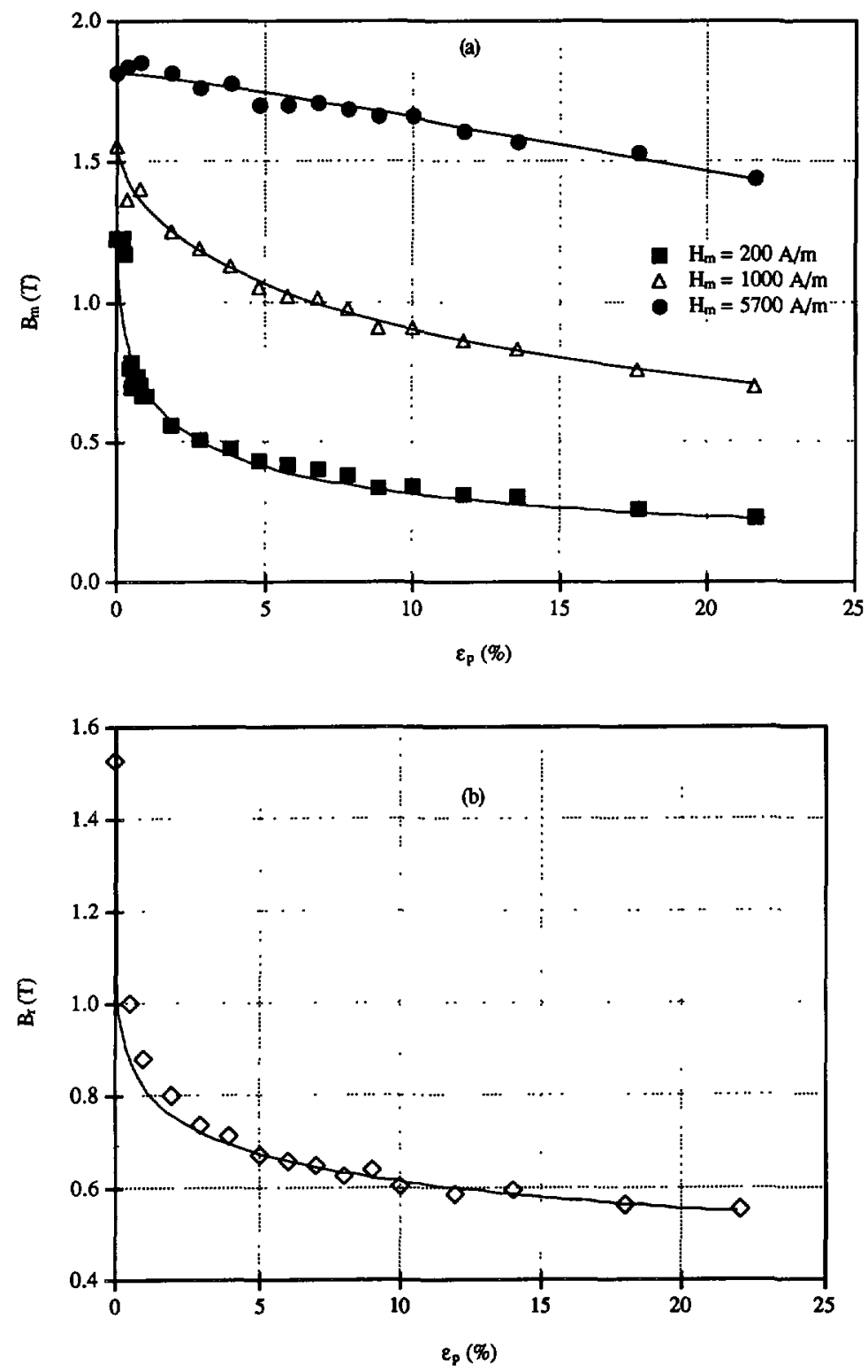

Fig. 5. - Evolution des caractéristiques magnétiques d'un Fe-3\% $\%$ Si non orienté avec les déformations plastiques. (a) $B_{\mathrm{m}}\left(\varepsilon_{\mathrm{p}}\right)$ pour différents $H_{\mathrm{m}}$, (b) $B_{\mathrm{r}}\left(\varepsilon_{\mathrm{p}}\right)$; (c) $P\left(\varepsilon_{\mathrm{p}}\right)$ pour différents $B_{\mathrm{m}}$, (d) $H_{\mathrm{c}}\left(\varepsilon_{\mathrm{p}}\right)$.

[Modifications of magnetic characteristics with plastic strains. (a) $B_{\mathrm{m}}\left(\varepsilon_{\mathrm{p}}\right)$ for various $H_{\mathrm{m}}$; (b) $B_{\mathrm{r}}\left(\varepsilon_{\mathrm{p}}\right) ;\left(\right.$ c) $P\left(\varepsilon_{\mathrm{p}}\right)$ for various $\left.B_{\mathrm{m}},(\mathrm{d}) H_{\mathrm{c}}\left(\varepsilon_{\mathrm{p}}\right) \cdot\right]$ 

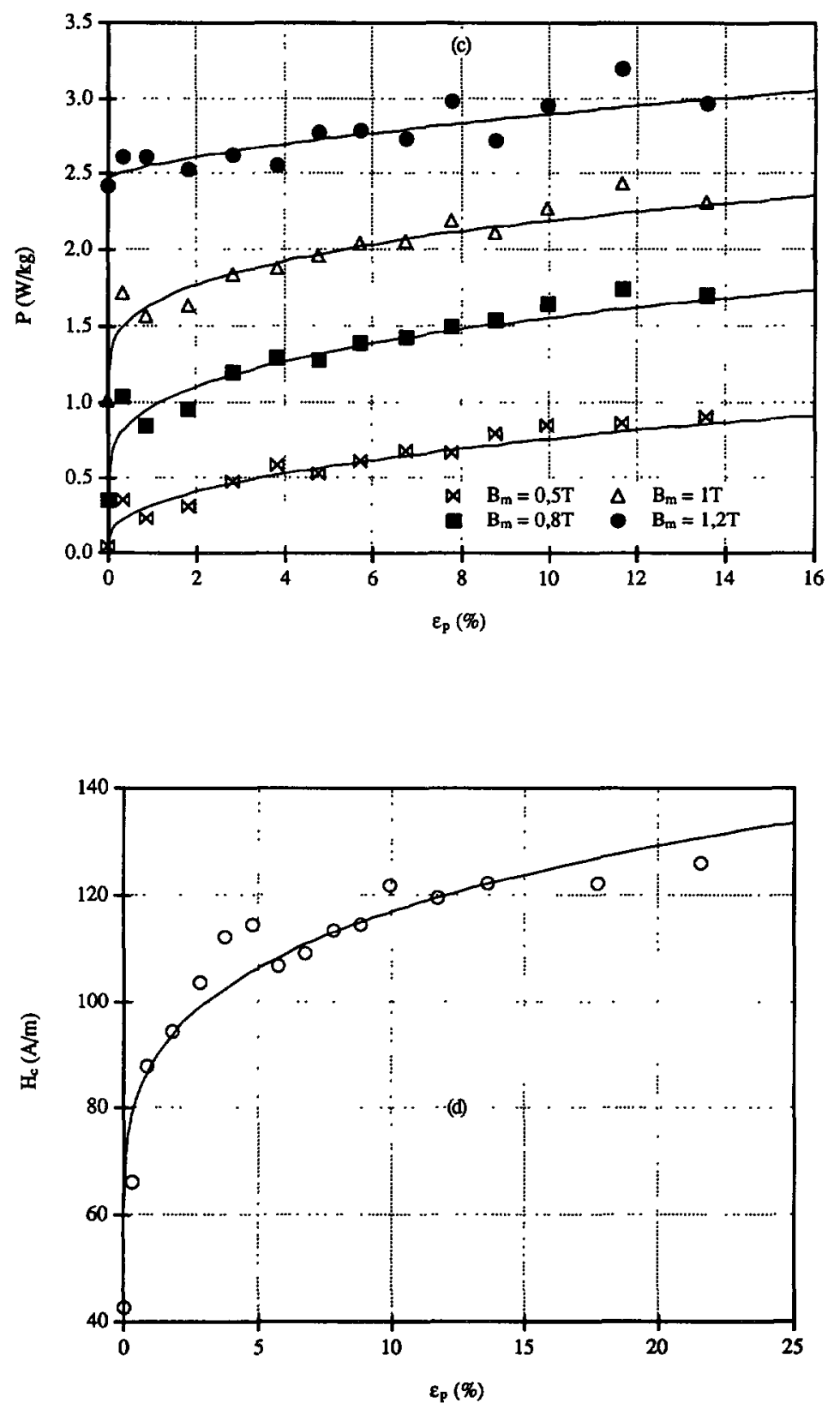

Fig. 5 (suite).

Le tableau III indique les différentes lois expérimentales reliant les caractéristiques magnétiques aux déformations plastiques.

2.3 EVOLUTION DE LA STRUCTURE DES DISLOCATIONS AVEC L'ÉCROUISSAGE. — L'examen par microscopie optique d'échantillons déformés sur le palier de Lüders révèle une structure géométrique ordonnée à l'intérieur de certains grains (Fig. 6), constituée de cellules, de forme 
Tableau III. - Modèles expérimentaux reliant les propriétés magnétiques aux déformations plastiques.

[Experimental models between magnetic properties and plastic strain.]

\begin{tabular}{|c|c|c|}
\hline & Formulation & Paramètres \\
\hline$B_{\mathrm{m}}$ & $\begin{array}{c}B_{\mathrm{m}}=B_{\mathrm{m}}^{0}\left(H_{\mathrm{m}}\right) \cdot \frac{k\left(H_{\mathrm{m}}\right)}{k\left(H_{\mathrm{m}}\right)+\varepsilon_{\mathrm{p}}^{n\left(H_{\mathrm{m}}\right)}} \\
B_{m}^{0}\left(H_{\mathrm{m}}\right): \text { courbe d'aimantation à } \varepsilon_{\mathrm{p}}=0 \% \\
k, n: \text { constantes caractéristiques du matériau, } \\
\text { fonctions de } H_{\mathrm{m}}\end{array}$ & $\begin{array}{c}k \in[1-200] \\
n \in[0,5-1,3] \\
H_{\mathrm{m}} \in[0-6000] \mathrm{A} / \mathrm{m}\end{array}$ \\
\hline$B_{\mathrm{r}}$ & $\begin{array}{c}B_{\mathrm{r}}=\frac{B_{\mathrm{r}}^{0}}{1+\varepsilon_{\mathrm{p}}^{n}} \\
B_{\mathrm{r}}^{0}: \text { rémanence à } \varepsilon_{\mathrm{p}}=0 \%\end{array}$ & $\begin{array}{l}B_{\mathrm{r}}^{0}=1,65 \mathrm{~T} \\
n=0.23\end{array}$ \\
\hline$\mu_{\mathrm{n}}$ & $\begin{array}{c}\mu_{\mathrm{r} 1}=\frac{\mu_{\mathrm{r} 1}^{0}}{1+\varepsilon_{\mathrm{p}}^{n}} \\
\mu_{\mathrm{r} 1}^{0}: \text { perméabilité relative initiale à } \varepsilon_{\mathrm{p}}=0 \%\end{array}$ & $\begin{array}{c}\mu_{\mathrm{r} 1}^{0}=2587 \\
n=0,6\end{array}$ \\
\hline$\mu_{\mathrm{rm}}$ & $\begin{array}{c}\mu_{\mathrm{rm}}=\mu_{\mathrm{rm}}^{0} \cdot \frac{\alpha}{\alpha+\varepsilon_{\mathrm{p}}^{n}} \\
\mu_{\mathrm{rm}}^{0}: \begin{array}{c}\text { perméabilité relative maximale à } \varepsilon_{\mathrm{p}}=0 \% \\
\alpha: \text { caractéristique du matériau }\end{array}\end{array}$ & $\begin{array}{c}\mu_{\mathrm{m}}^{0}=10850 \\
n=0,38\end{array}$ \\
\hline$P$ & $\begin{array}{c}P=P_{0} \cdot\left(1+k\left(H_{\mathrm{m}}\right) \cdot \varepsilon_{\mathrm{p}}^{n}\right) \\
P_{0}: \text { pertes totales de puissance initiales } \\
k, n: \text { constantes caractéristiques du matériau, } \\
\text { fonctions de } H_{\mathrm{m}}\end{array}$ & $\begin{array}{l}k \in[7,8-0,028] \\
\quad n \in[0,4-0,8] \\
B_{\mathrm{m}} \in[0-1,4] \mathrm{T}\end{array}$ \\
\hline$H_{\mathrm{c}}$ & $\begin{array}{c}H_{\mathrm{c}}=H_{\mathrm{c}}^{0} \cdot\left(1+\varepsilon_{\mathrm{p}}^{n}\right) \\
H_{\mathrm{c}}^{0}: \text { champ coercitif à } \varepsilon_{\mathrm{p}}=0 \%\end{array}$ & $\begin{array}{c}H_{\mathrm{c}}^{0}=43,5 \mathrm{~A} / \mathrm{m} \\
n=0,23\end{array}$ \\
\hline
\end{tabular}

quasi hexagonale, et de $26 \mu \mathrm{m}$ de taille moyenne. L'observation de lames minces par MET, prélevées sur ces mêmes échantillons, met en évidence des dislocations vis rectilignes du type $\{110\} a / 2\langle 111\rangle$ parallèles les unes aux autres (Fig. 7), ayant tendance à s'accumuler aux joints de grains. Au-delà du palier de Lüders, la structure hexagonale n'est plus observée. Entre 1 et $10 \%$ de déformation, la densité de dislocations augmente sensiblement, celles-ci étant réparties sous forme de petits amas de plus en plus denses (dimension typique de l'ordre de $0,1 \mu \mathrm{m}$ ) séparant des zones de métal où existent des dislocations isolées (Figs. $8 \mathrm{a}$ et $8 \mathrm{~b}$ ). A partir de $10 \%$, il se forme progressivement une structure très hétérogène constituée de murs de grande densité, de dimensions $(0,2 \mu \mathrm{m} \times 1 \mu \mathrm{m})$, délimitant des régions fortement appauvries en dislocations (Fig. 8c). 


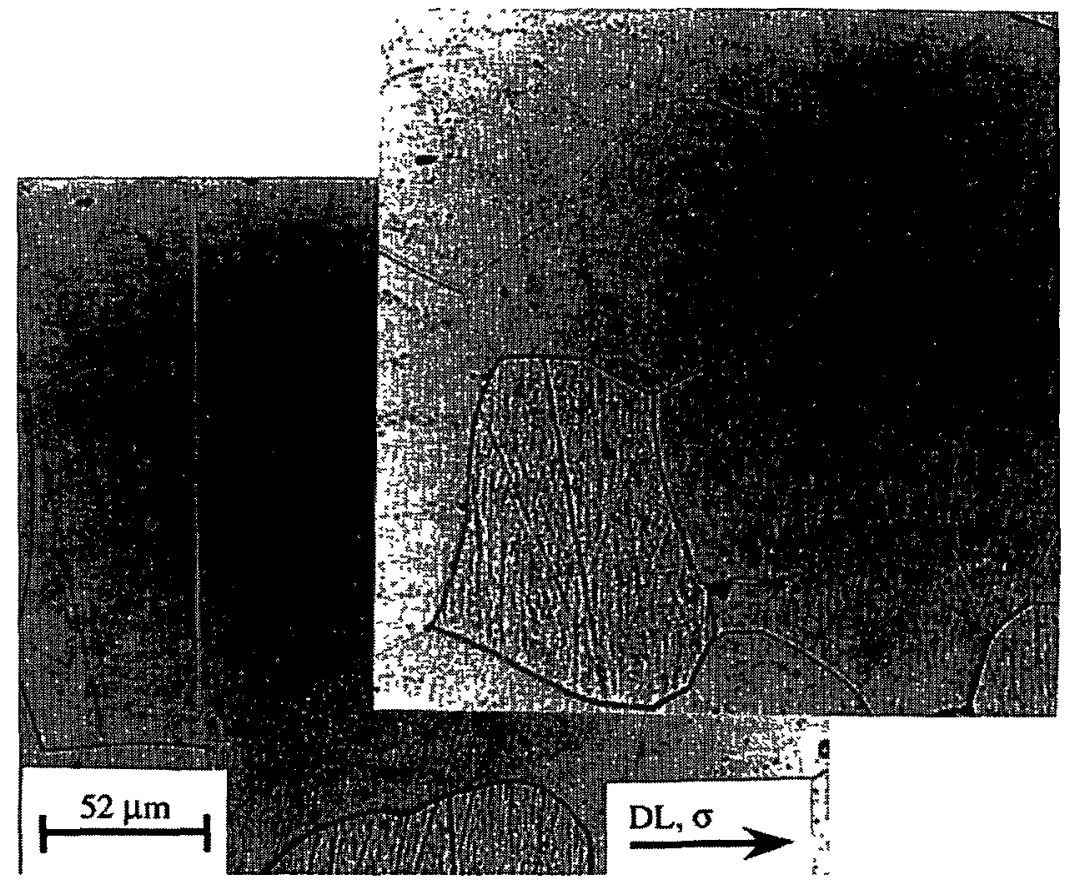

Fig. 6. - Micrographie optique d'un échantillon de Fe-3\%Si non orienté, déformé plastiquement de $0.34 \%$.

[Optical microphotographie of non-oriented $3 \% \mathrm{SiFe}$, strained up to $0.34 \%$.]

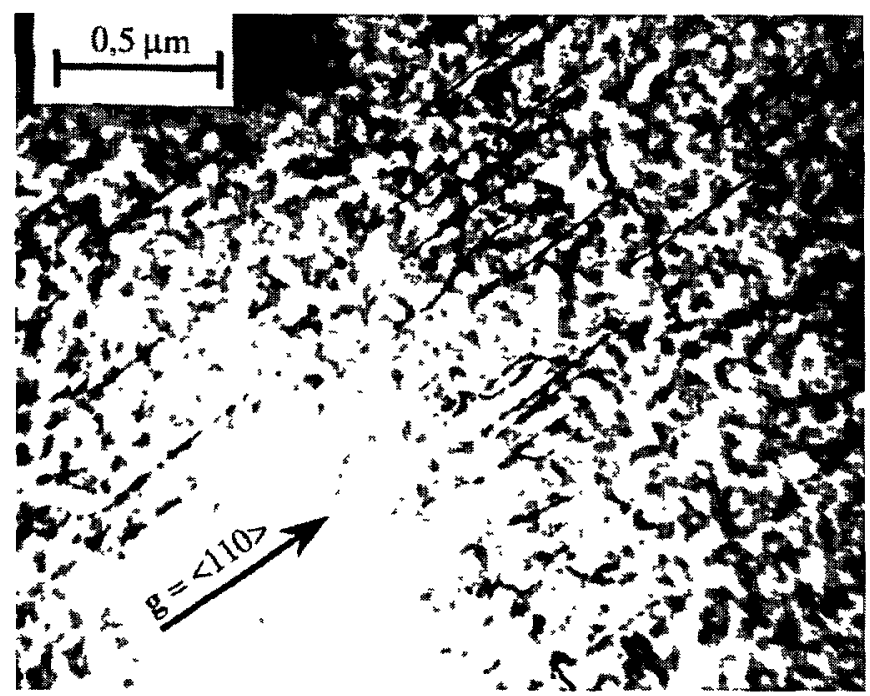

Fig. 7. - Observation par MET d'un échantillon de Fe-3\%Si non orienté déformé sur le palıer de Lüders, $\varepsilon_{\mathrm{p}}=0,34 \%$.

[TEM observation of non-oriented $3 \% \mathrm{SiFe}$. Lüders state. $\varepsilon_{\mathrm{p}}=0.34 \%$.] 


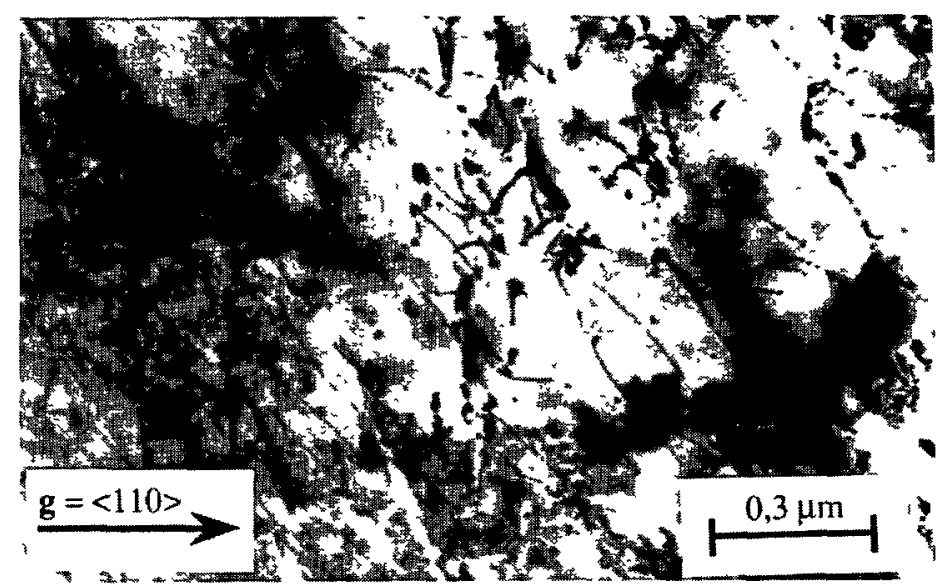

a)

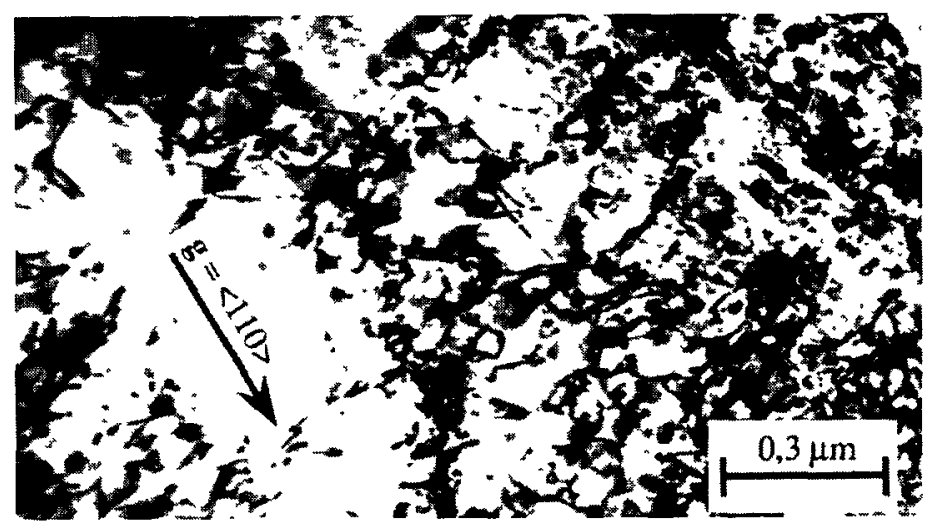

b).

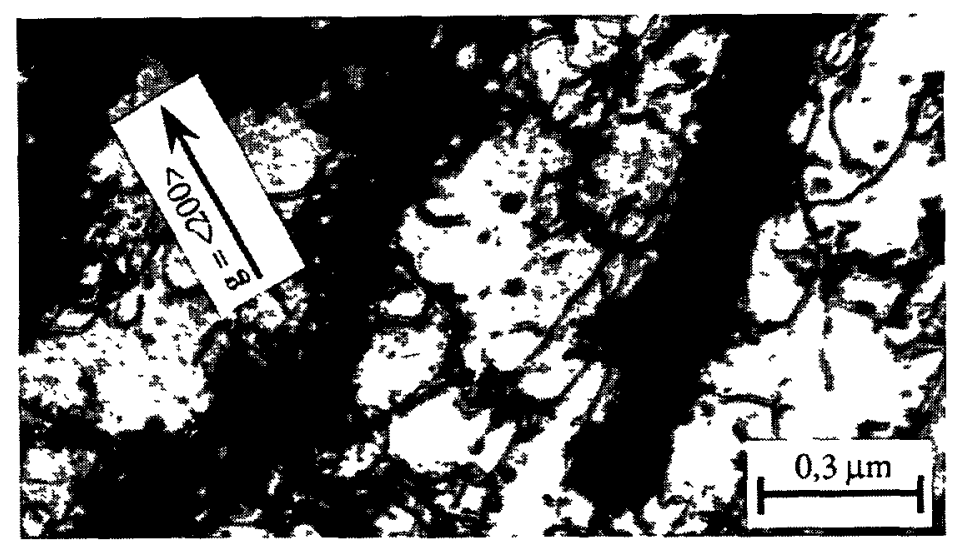

c)

Fig. 8. - Observation par MET d'un échantillon de Fe-3\%Si non orienté à différents niveaux de déformation plastique ; (a) $\varepsilon_{\mathrm{p}}=1,79 \%$; (b) $\varepsilon_{\mathrm{p}}=6,72 \%$; (c) $\varepsilon_{\mathrm{p}}=25,6 \%$.

[TEM observation of non-oriented $3 \% \mathrm{SiFe}$ with various plastic strain levels. (a) $\varepsilon_{\mathrm{p}}=1.79 \%$, (b) $\varepsilon_{\mathrm{p}}=6.72 \% ;(\mathrm{c}) \varepsilon_{\mathrm{p}}=25.6 \%$.] 
Entre 0 et $15 \%$ de déformation, la mesure de la densité de dislocations, réalisée par une méthode de comptage définie par Smith et Guthmann [18, 19] conduit à la loi expérimentale :

$$
\rho_{\mathrm{d}}=\rho_{\mathrm{d} 0} \cdot\left(1+\beta \cdot \varepsilon_{\mathrm{p}}\right)
$$

$\rho_{\mathrm{d} 0}$ étant la densité de dislocation initiale, estimée à $6 \times 10^{8} \mathrm{~cm} / \mathrm{cm}^{3}$, et $\beta$ une constante caractéristique. Pour des aciers à bas taux de carbone, Köster et Bangert [20] proposent la loi :

$$
\rho_{\mathrm{d}}=\alpha \cdot \varepsilon_{\mathrm{p}}^{0.5}
$$

pour une déformation de laminage comprise entre 0 et $70 \%$. Nous avons représenté sur la figure 9 nos points expérimentaux et le modèle de Köster et Bangert. Compte tenu de la forte dispersion sur $\rho_{\mathrm{d}}$ (de l'ordre de $70 \%$ ), la densité de dislocations de l'alliage Fe $3 \%$ Si non orienté évolue avec les déformations plastiques conformément aux aciers doux standards.

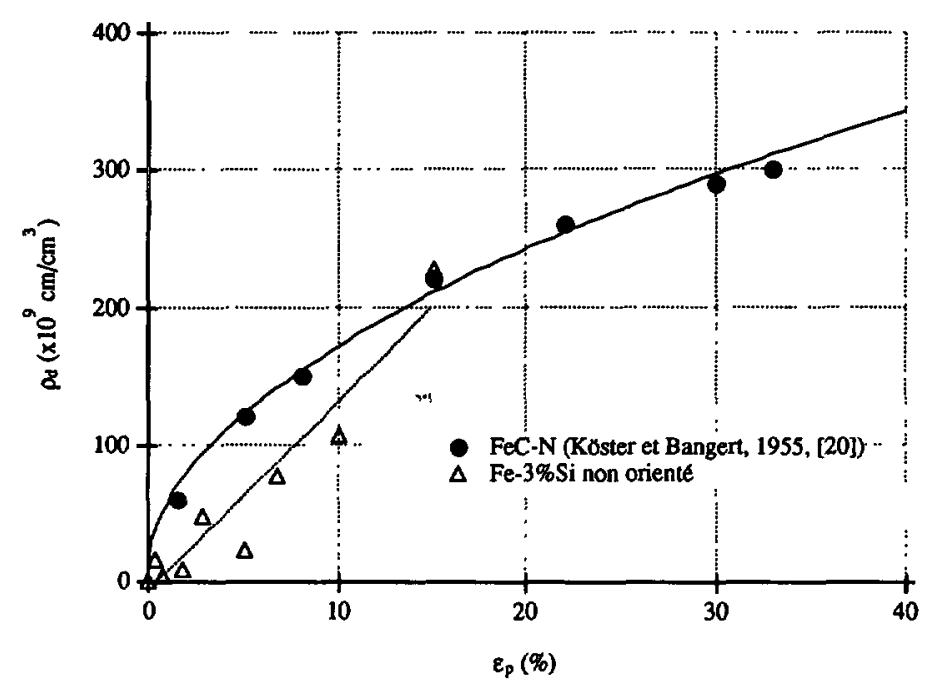

Fig. 9. - Evolution de la densité de dislocations du $\mathrm{Fe}-3 \% \mathrm{Si}$ non orienté et comparaison avec les résultats obtenus par Köster et Bangert pour un acier faiblement allié.

[Effect of plastic strains on dislocation density for non-oriented $3 \% \mathrm{SiFe}$ and results of Köster and Bangert concerning a low-alloyed steel.]

\section{Discussion.}

3.1 APPliCATION DU MOdÈle DU POTENTIEL CONSERVATIF. - Le modèle du potentiel conservatif stipule qu'une paroi reste rigide et que son mouvement est influencé par les fluctuations locales de l'énergie potentielle du matériau au voisinage de sa position, du fait de l'existence d'hétérogénéités [21,22]. L'application de ce modèle au cas réel d'un alliage contenant un nombre important de défauts a été effectuée par de nombreux auteurs. En particulier. Kronmüller et al., par un calcul théorique basé sur le traitement statistique du mouvement des parois, montrent que le produit $\mu_{\mathrm{r}} . H_{\mathrm{c}}$ doit demeurer constant, et que 
$\mu_{\mathrm{r}}$ et $H_{\mathrm{c}}$ évoluent en fonction de la densité de dislocations $\rho_{\mathrm{d}}$ de la façon suivante [23-25] :

$$
\begin{aligned}
& H_{\mathrm{c}}=\text { Cte } \cdot \rho_{\mathrm{d}}^{1 / 2} \\
& \mu_{\mathrm{r} 1}=\text { Cte } \cdot \rho_{\mathrm{d}}^{-1 / 2}
\end{aligned}
$$

L'existence d'une relation bijective entre $\rho_{d}$ et $\varepsilon_{p}$ dans les alliages de fer doux, telle que nous l'avons déterminée, implique que dans le cadre du modèle du potentiel conservatif, nous devons vérifier :

$$
H_{\mathrm{c}} \cdot \mu_{\mathrm{rl}}=\text { Cte }, \quad \forall \varepsilon_{\mathrm{p}} .
$$

La figure 10 présente l'évolution de $H_{\mathrm{c}} \cdot \mu_{\mathrm{r} 1}$ avec $\varepsilon_{\mathrm{p}}$ pour le Fe-3\%Si non orienté. Le produit diminue très rapidement dès les faibles déformations. Plus précisément, $H_{c} \cdot \mu_{\mathrm{r} 1}$ évolue en deux stades grossièrement linéaires: forte diminution entre 0 et $10 \%$ de déformation, relative stagnation pour $\varepsilon_{\mathrm{p}}>10 \%$. Le modèle du potentiel conservatif pourrait alors être valide dans la gamme des fortes déformations. Cependant, des observations microscopiques de la configuration en domaines magnétiques d'alliages $\mathrm{Fe}-3 \% \mathrm{Si}$ à grains orientés fortement écrouis montent une forte courbure des parois principales sous l'effet du pouvoir d'ancrage important des murs de dislocations [14]. Des observations analogues ont été réalisées sur du fer polycristallin de haute pureté [26]. Une des hypothèses de base du modèle n'est donc plus vérifiée.

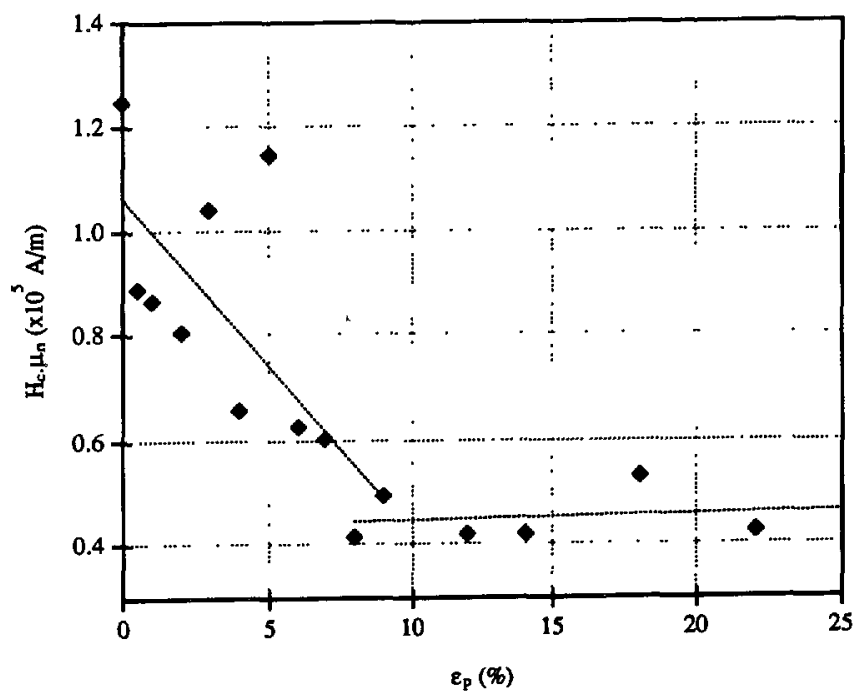

Fig. 10. - Evolution du produit $H_{\mathrm{c}} \cdot \mu_{\mathrm{r}}$ avec les déformations plastiques.

[Modifications of the product $H_{\mathrm{c}} \cdot \mu_{\mathrm{r}}$ with plastic strains.]

Le modèle du potentiel conservatif n'est donc pas satisfaisant pour décrire les interactions entre parois et dislocations dans les alliages polycristallins $\mathrm{Fe}-3 \% \mathrm{Si}$ non orientés. Ce résultat rejoint ceux de Astié et Degauque pour le fer de haute pureté [26].

3.2 APPLICATION DU MODÈLE DE COURBURE DES PAROIS. - L'existence de dislocations au sein de la matrice métallique peut provoquer l'ancrage des parois de Bloch. Sous l'action d'un 
champ magnétique extérieur, leur mouvement est perturbé: la variation d'aimantation s'effectue alors par déplacement des parties libres de la paroi qui perd ainsi son caractère plan [27]. Le mouvement irréversible se réalise pour un rayon de courbure critique fonction de la distance moyenne entre obstacles [28]. L'application de ce modèle au cas réel d'une densité de défauts $\rho_{\mathrm{d}}$ permet d'obtenir la relation de proportionnalité [29] :

$$
H_{\mathrm{c}}=\mathrm{Cte} \cdot \rho_{\mathrm{d}}^{2 / 3}
$$

L'exposant $2 / 3$ est proche de $1 / 2$, exposant caractéristique du modèle du potentiel conservatif. La précision de nos mesures sur $\rho_{\mathrm{d}}$ ne nous permet donc pas de trancher. Cependant, nous pouvons faire les observations suivantes : en début de déformation, le nombre de dislocations mobiles entre les amas est important. Au cours de l'aimantation du matériau, le déplacement d'une paroi peut entraîner dans son voisinage le mouvement de ces dislocations, par analogie aux observations réalisées sur des mesures de microfluage par Allen et Donowan [30]. D'autre part, dans le domaine des grandes déformations, la densité importante des dislocations fixes et leur répartition fortement hétérogène en murs (Fig. 8c) ancrent et courbent les parois principales très facilement [14]. Le modèle de courbure des parois semble donc plus approprié pour l'interprétation de nos résultats expérimentaux. La différence de comportement significative intervenant avant et après $8 \%$ de déformation pourrait alors s'expliquer en termes d'intensité de force de blocage des parois sur les obstacles.

3.3 ETAT DE CONTRAINTE INTERNE DU FeSi. - Les paramètres d'écrouissage, déterminés précédemment en fonction de $\varepsilon_{\mathrm{p}}$, permettent d'accéder à l'état de contrainte interne après déformation plastique et décharge. L'étude du spectre de diffraction des rayons $\mathrm{X}$ d'échantillons déformés nous indique une faible translation des pics et un élargissement sensible à mesure que croît la déformation plastique. Celà signifie que les contraintes internes macroscopiques n'évoluent pas ; seules augmentent avec l'écrouissage les microdéformations résiduelles dues aux dislocations.

Les mesures de $R$ et $X$ permettent de préciser l'évolution des contraintes internes macroscopiques. Nous pouvons raisonner sur l'évolution de la surface de charge du matériau au cours de l'écrouissage (hypothèse du critère de plasticité de Von Misès) (Fig. 11). A l'état initial, $R$ et $X$ sont supposés nuls : la surface de charge est alors représentée par un cercle ayant pour rayon la limite d'élasticité initiale $\sigma_{\mathrm{e}}$ et centré sur l'axe des contraintes (point $\mathrm{O}$ ). Au cours de l'écrouissage, nous avons une composante cinématique et une composante isotrope : la surface se déplace alors dans l'espace des contraintes, tout en se contractant, $R$ étant négatif. Le centre de la surface de charge se déplace alors de $O$ à $O^{\prime}$. Lorsque nous déchargeons l'éprouvette après avoir atteint un certain taux de déformation plastique, le centre de la surface de charge vient se positionner en $\mathrm{O}^{\prime \prime}$, état mécanique de l'éprouvette au moment où nous réalisons les mesures magnétiques. Nous avons alors $X=\left(R+\sigma_{\mathrm{e}}\right) . R$ représente donc dans notre configuration l'état de contraintes internes du matériau à grande échelle, après déformation plastique. La figure 4 montre une variation très faible de $R$ avec $\varepsilon_{\mathrm{p}}$, une fois dépassé le palier de Lüders. Par conséquent, les contraintes internes macroscopiques dans le matériau, après écrouissage et décharge, restent constantes.

La définition adoptée ici pour la contrainte interne $\sigma_{1}$ diffère de celle de Astié et al. qui, par des mesures d'amortissement magnétomécanique, déterminent une évolution en trois stades linéaires de $\sigma_{1}$ avec $\Delta \sigma=\sigma-\sigma_{\mathrm{e}}$, « contrainte plastique pure » appliquée au matériau [31]. La contrainte imposée en chaque instant lors de l'essai de traction s'écrit :

$$
\sigma=\sigma_{\mathrm{e}}+R+X \Rightarrow \Delta \sigma=R+X
$$

La contrainte $\sigma_{1}$ mesurée par Astié et al. est donc représentative de l'écrouissage total 


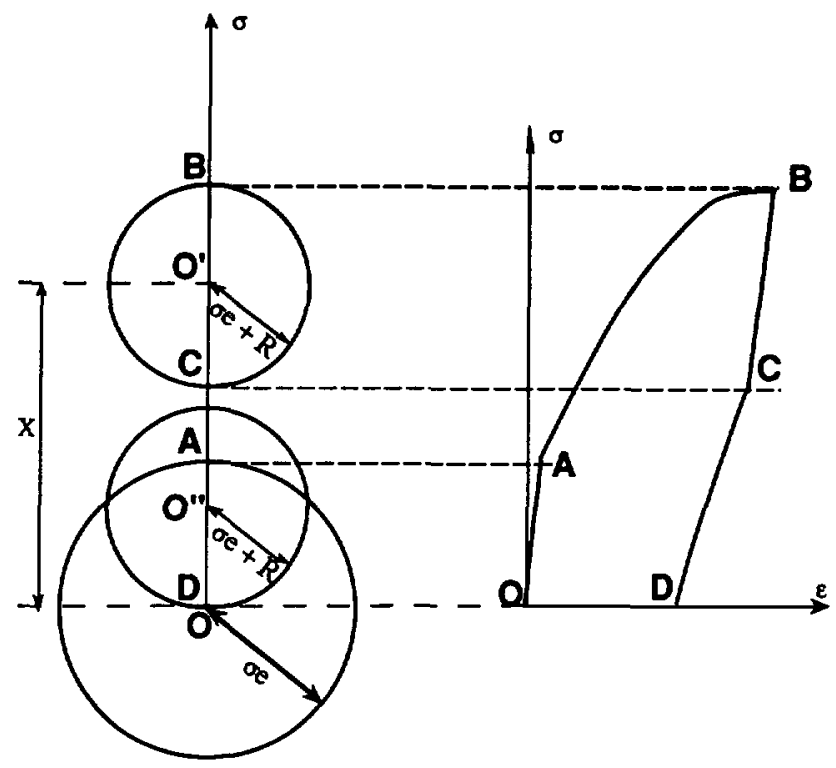

Fig. 11. - Evolution schématique de la surface de charge au cours d'un essai de charge-décharge.

[Schematic evolution of the loading surface in the course of loading-unloading test.]

$(R+X)$ mais pas de la contrainte interne existant dans le matériau après l'essai de traction. Dans le cas d'un fer polycristallin de haute pureté, l'existence de trois configurations principales en dislocations (dislocations isolées à faibles déformations; petits amas aux déformations moyennes et murs denses de dislocations aux fortes déformations [32]) peut être reliée à l'évolution du champ coercitif avec $\Delta \sigma$ [33]. La figure 12 montre l'évolution de $H_{\mathrm{c}}$ avec $\Delta \sigma$ et donne pour comparaison les résultats obtenus sur du fer de haute pureté par Degauque. La courbe $H_{\mathrm{c}}(\Delta \sigma)$ du matériau NO ne présente que deux stades linéaires : brusque montée entre 0 et $1 \%$ de déformation (correspondant à un $\Delta \sigma$ quasiment nul caractéristique du palier de Lüders), suivie d'une croissance plus lente par la suite. Le premier stade présent pour le fer de haute pureté, où $H_{c}$ reste constant sur une gamme importante de $\Delta \sigma$, n'existe pas pour l'alliage non orienté.

Ce résultat indique que dès le tout début de déformation, lorsque nous sommes sur le palier de Lüders, la répartition des dislocations n'est pas homogène. L'existence de cellules hexagonales confirme ce résultat. Ceci est en accord avec les résultats de Iricibar et al. (cité dans [34]) qui mettent en évidence l'existence d'une multiplication catastrophique du nombre de dislocations mobiles au cours de la propagation du palier de Lüders. Lorsque le palier est entièrement parcouru, $\Delta \sigma$ commence à croître et $H_{\mathrm{c}}$ augmente plus lentement.

\section{Conclusion - perspectives.}

Les caractéristiques magnétiques d'alliages $\mathrm{Fe}-3 \% \mathrm{Si}$ NO subissent une profonde détérioration entre 0 et $5 \%$ de déformation plastique ; cet effet est très sensible aux faibles et moyennes amplitudes du champ magnétique imposé, puis s'estompe à mesure que nous tendons vers la saturation magnétique. L'analyse microscopique met en évidence une structure en dislocations fortement hétérogène pour la gamme de déformation représentative du palier de Lüders. Le début de l'écrouissage, survenant à la fin du palier, est caractérisé par la formation progressive de petits amas séparant des zones de matériau où existent des dislocations isolées. A mesure 


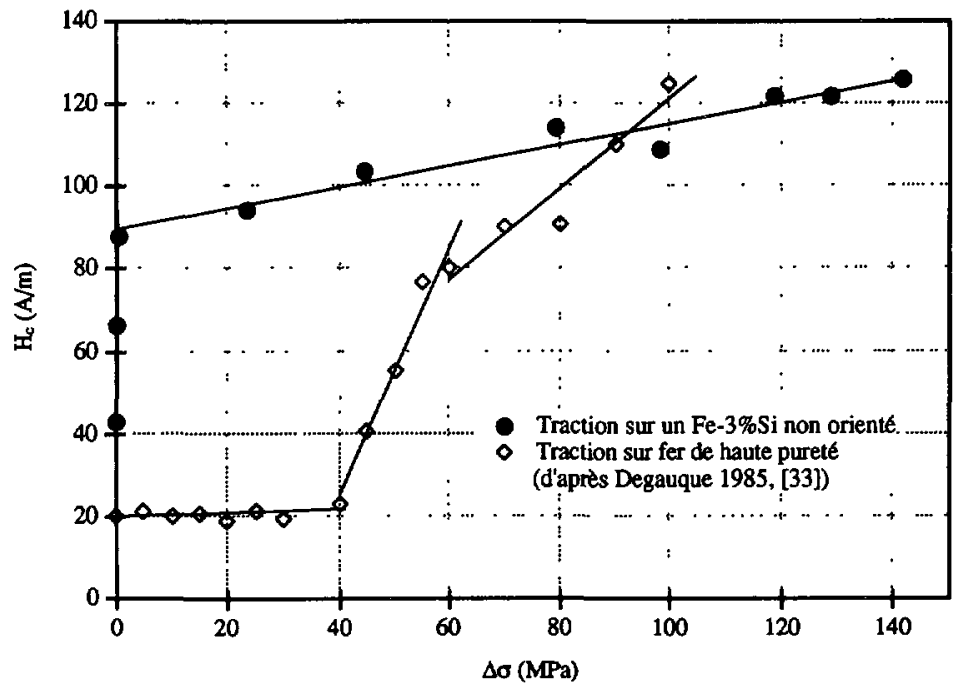

Fig. 12. - Variation du champ coercitif avec la contrainte plastique pure pour le $\mathrm{Fe}-3 \%$ Si non orienté et pour un fer polycristallin de haute pureté.

[Coercive field versus plastic stress for non-oriented $3 \% \mathrm{SiFe}$ and a polycristalline high-purity iron.]

qu'augmente la déformation, ces amas deviennent de plus en plus dense, et finissent par former des murs délimitant des régions fortement appauvries en dislocations. La structure la plus nocive est donc celle créée au cours du palier de Lüders puis les petits amas faiblement denses. En revanche, la configuration en murs denses ne modifie que substantiellement les caractéristiques magnétiques.

L'interprétation de ces différentes observations semble pouvoir être réalisée dans le cadre d'un modèle de courbure de parois. Le paramètre contrôlant les phénomènes d'interactions magnétomécaniques est dans notre cas la déformation plastique ou la densité et la structure des dislocations, plutôt que l'état de contraintes internes macroscopiques régnant au sein du matériau après écrouissage.

L'étude des dégradations des caractéristiques magnétiques des alliages FeSi avec les déformations plastiques trouve une application directe dans l'influence de la mise en forme des tôles sur les propriétés magnétiques de structures électromécaniques complexes. Des mesures de microdureté au voisinage du passage d'un outil de poinçonnage permettent d'atteindre le gradient de déformation des zones de métal affectées $[14,35]$. Les corrélations de ces mesures avec les relations empiriques reliant les différents paramètres magnétiques aux déformations permet d'obtenir des cartes tridimensionnelles du type $B(H, d)$ ou $P(B, d), d$ étant la distance au bord de découpe. Ces résultats peuvent également être appliqués avec profit à la détermination numérique des pertes de puissance, par éléments finis, d'une machine électrique [36].

\section{Bibliographie}

[1] Ille J. L., Mouillet A., Cottet J. J., Eck A., Influence des hautes fréquences 'et des hautes températures sur les pertes fer d'une machine électrique ; journées d'études sur l'électrotechnique avancée. DRET, Paris (1989). 
[2] Allano S., Lebouc A., Perret R., Pertes dans les circuits magnétiques : une modélisation complexe ; RGE, n 3 (mars 1987).

[3] Craik D. J., Wood M. J., Magnetization changes induces by stress in a constant field, $J$ Phys. $D$ : Appl. Phys. 3 (1970) 1009.

[4] Moses D., Influence of compressive stress on magnetic properties of commercial (110) [001] oriented silicon-iron, IEEE Trans. Magn. 16 (1980) 454.

[5] Dobranski L. G., Jiles D. C., Atherton D. L.. Dépendence of the anhysteretic magnetization on uniaxial stress in steel, J. Appl. Phys. 57 (1985) 4229.

[6] Atherton D. L., Jiles D. C., Effects of stress on magnetization, NDT international 19 (1986) 15.

[7] Sablik M. J., Kwun H., Burkhardt G. L., Jiles D. C., Model for the effect of tensile and compressive stress on ferromagnetic hysteresis, J. Appl. Phys. 61 (1987) 3799.

[8] Pitman K. C., The influence of stress on ferromagnetic hysteresis, IEEE Trans. Magn. 26 (1990) 1978.

[9] Shirkoohi G. H., Moses A. J., Effects of stress on iron loss and apparent power in low silicon nonoriented electrical steel, An. Fis., serle B 86 (1990) 265.

[10] Bozorth R. M., Ferromagnetsm (D. Van Nostrand Company, 1951).

[11] Neurath P. W., Effect of plastic and elastic stresses on the losses and the domain configurations of grain-oriented 3pct Si-Fe, J. Metals, Transactions AIME (1956) 1319.

[12] Stodolny J., Groyecki J., Gorczyca S., Lampa H.. Structure, plasticity and magnetic properties of FeSiAl alloys, An. Fis, serie B 86 (1990) 241.

[13] Matsumura K.. Fukuda B., Recent developments of non-oriented electrical steel sheets, IEEE Trans. Magn. 20 (1984) 1533.

[14] Hug E.. Etude des dégradations des propriétés magnétiques d'alliages $\mathrm{Fe}-3 \% \mathrm{Si}$ avec les déformations plastiques. Application à la mise en œuvre des tôles utilisées dans la conception des moteurs électriques; Thèse de doctorat, UTC (1993).

[15] Pilvin P., Approches multiéchelles pour la prévision du comportement anélastique de métaux; Thèse de doctorat, Paris VI (1990).

[16] Lemaitre J., Chaboche J. L.. Mécanique des matériaux solides, $2^{\mathrm{e}}$ édıtion (Dunod, Bordas, 1988).

[17] Qureshi A. H., Chaudary L. N., Influence of plastic deformation on coercive field and initial susceptibility of Fe-3,25\%Si alloys, J. Appl. Phys. 41 (1970) 1042.

[18] Hirsch P. B., Howie A., Nicholson R. B., Pashley D. W., Whelan M. J., Electron microscopy of thin crystals, $2^{\text {nd }}$ edition (London, Butterworths, 1967).

[19] Dumas E., Hug E., Clavel M., Ille J. L., Etude du comportement magnétique d'alliages Fe-3\%Si non orientés utilisés dans la conception de machines électriques, J. Phys. IV France 2 (1992) C3-47.

[20] Koster W., Bangert L., Bestimmung der Versetzungliniendichte von verformten Eisen, Acta Met, n 3 (1955) 274.

[21] Chen C. W., Magnetism and metallurgy of soft magnetic materials (North-Holland publishing company, 1977).

[22] Chikazumi S., Physics of magnetism (John Wiley \& sons, Inc., 1964).

[23] Seeger A., Kronmuller H., Rieger H., Trauble H., Effect of lattice defects on the magnetization curve of ferromagnets, J. Appl. Phys. 35 (1964) 740.

[24] Kronmuller H., Magnetic techniques for the study of dislocations in ferromagnetic materials, International $J$ Nondestructive Testing 3 (1972) 315.

[25] Kronmuller H., Statistical theory of Rayleigh's law, Z. Angew. Phys. 30 (1970) 9.

[26] Astie B., Degauque J., Porteseil J. L., Vergne R., Predictions of the random potential energy models of domain wall motion : an experimental investigation on high-purity iron, J.M.M.M., n 28 (1982) 149.

[27] Dietze H. D., Theorie der Blochwandwölbung mit Streufeldeinfluß, Z. Phys. 149 (1957) 276.

[28] Jiles D., Introduction to magnetism and magnetıc materials (Chapman \& Hall, 1991).

[29] Labusch R., Calculation of the critical field gradient in type II superconductors, Crystal lattice defects 1 (1969) 1.

[30] Allen C. W., Donovan J. A., Magnetoelastic interaction of dislocations and ferromagnetic domain 
walls and torsional microcreep experiments on iron and nickel tubes, J. Appl. Phys. 38 (1967) 1329.

[31] Astie B., Degauque J., Détermination par amortissement magnétomécanique de la répartition des contraintes internes dans le fer pur écroui, J. Phys. Colloq. France 44 (1983) C9-461.

[32] Taylor R. A., Jakubovics J. P., Astie B., Degauque J., Direct observation of the interaction between magnetic domain walls and dislocations in iron, J. Magn. Magnetic Mater. 31-34 (1983) 970.

[33] Degauque J., Les pertes d'énergie dans les ferromagnétiques métalliques doux : origines physiques ; Mémoires et études scientifiques, revue de métallurgie (janvier 1985) p. 5.

[34] Strudel J. L., Interaction des dislocations avec des impuretés mobiles ; dislocations et déformation plastique, école d'été d'Yravals (1979) p. 199.

[35] Hubert O., Hug E., Influence of plastic strain on the non-oriented $3 \%$ silicon-iron magnetic behaviour. Application to a manufacturing test by punching, article soumis à Mat. Sci. Technol. (1993).

[36] Hug. E., Dumas F., Biedinger J. M., Clavel M., Influence des déformations plastiques sur le comportement magnétique d'alliages fer-silicium, à paraître dans Mémoires et études scientifiques, revue de Métallurgie. 